\title{
Measurement of ice thickness on vitreous ice embedded cryo-EM grids: investigation of optimizing condition for visualizing macromolecules
}

\author{
Hye-Jin Cho ${ }^{\dagger}$, Jae-Kyung Hyun ${ }^{\dagger}$, Jin-Gyu Kim, Hyeong Seop Jeong, Hyo Nam Park, Dong-Ju You
} and Hyun Suk Jung ${ }^{*}$

\begin{abstract}
Background: Cryo-electron microscopy is an excellent method for the structural analysis of biological materials. Advantage of its use over conventional electron microscopy techniques is the preservation of the sample in a near-native, hydrated state. To achieve the analysis with greatly improved structural details, optimization of various parameters involved in sample vitrification is required. Most considerable parameter is the thickness of ice: thick and thin layers are ideally in favor for larger and smaller target objects.

Findings: We measured the thickness of vitreous ice from different types of widely used holey carbon grids using cryo-EM and electron energy loss spectroscopy. It showed that Quantifoil grids are suitable for the structural analysis of large biological macromolecules (>100 nm in size), whereas the use of lacey and C-flat grids are ideal for smaller particles.

Conclusions: This report provides informative details that may help increasing chances of obtaining optimal vitreous ice for various biological objects with different sizes, hence facilitate the successful application of cryo-electron microscopy.
\end{abstract}

Keywords: Transmission electron microscopy, Cryo-electron microscopy, Vitreous Ice thickness

\section{Findings}

Transmission electron microscopy (TEM) is a powerful and versatile technique that enables direct visualization of biological samples of sizes ranging from whole cell to near-atomic resolution details of a single protein molecule (Frank 2006). For structural analysis of protein macromolecular assemblies (e.g. virus particles) and proteins that harbor multiple conformations (e.g. ribosome), TEM has evolved into a popular tool despite lower achievable resolving power than X-ray crystallography and nuclear magnetic resonance (NMR) spectroscopy. Major advantages of TEM over other structure determination techniques include minimization of artifacts

\footnotetext{
* Correspondence: hyunsukjung@kbsi.re.kr

${ }^{\dagger}$ Equal contributors

Division of Electron Microscopic Research, Korea Basic Science Institute, 113 Gwahangno, Daejeon 305-333, Korea
}

derived from harsh crystallization conditions, direct visualization of proteins in near-physiological environment, and relatively larger molecular mass limit for the analysis (van Heel et al. 2000). In addition, relatively small amount of protein sample is required for the TEM analysis, and therefore overcomes limited availability of target macromolecules.

Among many specialized TEM techniques, cryoelectron microscopy (cryo-EM) allows for direct imaging and structural analysis of fully hydrated biological specimen in near-physiological environment (Frank 2006). The technique involves preservation of the sample in vitrified aqueous solution (Adrian et al. 1984; Cavalier et al. 2008) and therefore prevents artifacts that can be derived from chemical fixation, staining and dehydration (Frank 2006; van Heel et al. 2000). Moreover, in comparison to stain-embedded samples of which the resolution is 
limited to the size of heavy metal salts, cryo-EM can achieve structure determination of macromolecular assemblies at near-atomic resolution because the image formation directly results from electron scattering of biological specimen (Gonen et al. 2005). Inherently low contrast of cryo-EM images can be compensated by using image processing tools which greatly improve signal-to -noise ratio though image alignment and averaging (Frank et al. 1996; van Heel et al. 1996; Grigorieff 2007). Despite these advantages, cryo-EM is often technically demanding. One of the difficulties comes from preparation of vitrified specimen that is suitable for efficient data collection and high-resolution image processing. An ideal specimen (a) should be free of contaminants such as ethane and ice crystals, (b) should have maximum number of particles within a field of view, yet the particles are sufficiently spaced, and (c) has a vitrified solution that is thick enough to contain the protein particles but thin enough to prevent excessive beam interference that reduces image contrast (Orlova \& Saibil 2011). Therefore, for a given macromolecular sample, both concentration and ice thickness need be optimized by an experienced researcher through trialand-error.

Because specimen preparation for biological cryo-EM is critical for achieving high-resolution structure determination, understanding relationship between the practical use of instrumentation and the materials is important. In order to entrap biological specimen in vitreous ice that is free of supporting material, EM grid coated with a thin carbon film with perforated holes, namely "holey carbon grid", is used. Uniformity of sample adsorption and even ice thickness across the EM grid requires the surface of carbon film to be rendered hydrophilic, which is usually achieved by glow discharging in vacuum (Aebi \& Pollard 1987). After loading aqueous sample onto a holey carbon grid, excess solution is blotted and the grid is plunged into cryogen that is cooled to liquid nitrogen temperature. This step is crucial for reproducible and consistent outcome of the experiment, which is aided by using automated vitrification devices (Iancu et al. 2006; Grassucci et al. 2008). In addition, such devices can reduce ice crystal contamination since manual handling of the sample is minimized. In combination with user-defined virification conditions, a principal step to find the optimal thickness of vitreous ice is the choice of a particular type of support film.

In this study, using cryo-EM and electron energy loss spectroscopy (EELS), we measure one of critical but basic parameter for successful application of cryo-EM, which is the thickness of vitreous ice from various types of holey carbon grids that are commercially available. The results suggest that larger and smaller biological objects are ideally suitable for the use of Quantifoil and C-flat grids (Lacey grids), respectively, and the thickness of ice in each type of grid is dependent on the thickness of carbon support film.

\section{Materials and methods}

\section{Specimen preparation}

Three types of widely used holey carbon grids were subjected to the experiments; (a) Quantifoil grid $(\mathrm{Cu} 300$ mesh, R2/2, SPI Supplies, U.S), (b) Lacey carbon grid (Agar Scientific, U.K.) and (c) C-flat (Cu 200 mesh, CF1.2/1.3, Protochips Inc., U.S.). The grids were rendered hydrophilic using plasma cleaner (PDC-32G-2, Harrick Plasma, U.S), followed by application of $5 \mu \mathrm{l}$ of distilled water. The sample grid was semi-automatically vitrified using Vitrobot Mark I (FEI, U.S), at 100\% relative humidity and $4{ }^{\circ} \mathrm{C}$. Blot time of 3.5 seconds and blot offset of $-0.5 \mathrm{~mm}$ were consistently applied to all viritirification processes. The entire procedure is described in (Grassucci et al. 2008).

\section{Cryo-electron microscopy}

Vitrified samples were maintained at liquid nitrogen temperature during sample transfer and image acquisition using a cryo transfer holder (cryo holder-626, Gatan, U.S). The sample temperature throughout the experiment was carefully monitored using SmartSet Controller (Gatan, U.S), where the temperature was typically kept at approximately $-177^{\circ} \mathrm{C}$ within the electron microscope. Tecnai $\mathrm{G}^{2}$ Sprit Twin equipped with lanthanum hexaboride $\left(\mathrm{Lab}_{6}\right)$ gun operating at $120 \mathrm{kV}$ was used to acquire images. Micrographs were recorded using Ultracan $^{\text {Tu }} 4000$ CCD detector (Gatan, U.S), under low dose imaging mode, with typical electron does of $10-20 \mathrm{e} / \AA^{2}$.

\section{Electron energy loss spectroscopy}

Ice thickness was estimated using log-ratio method (Malis et al. 1998), which is based on following relationship:

$$
{ }^{t} / \lambda_{\rho}=\ln \left(\frac{\mathrm{I}_{\mathrm{t}}}{\mathrm{I}_{0}}\right)
$$

where $t$ is the specimen thickness, $\lambda_{\rho}$ is the effective mean free path length for inelastic scattering, and $I_{0}$ and $I_{t}$ are zero-loss peak in electron energy loss spectrum and the whole spectrum, respectively. Electron energy loss spectrum was obtained using Gatan Imaging Filter (GIF) system (T12, Gatan, U.S), and the objective aperture that corresponds to the collection angle of $1.639 \mathrm{mrad}$ was used for data collection. Integrated ratio between $I_{t}$ and $I_{0}$ was used for estimation of inelastic mean free path, from which the specimen thickness was calculated using Digital Micrograph 3.0 software (Gatan, U.S). Typically, EELS was performed at nominal magnifications of $\times 28,000$ and $\times 68,000$, suitable for obtaining the spectrum of the entire hole and the center of vitreous ice, respectively. 


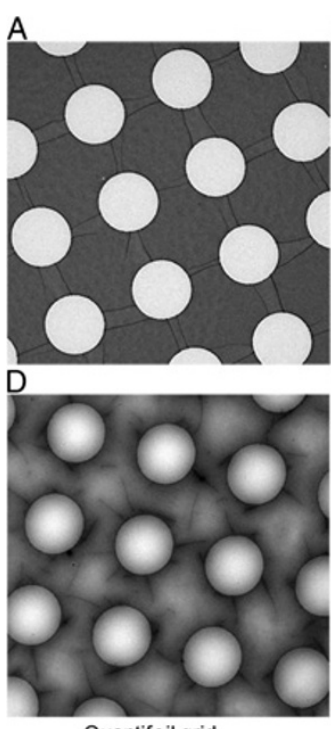

Quantifoil grid

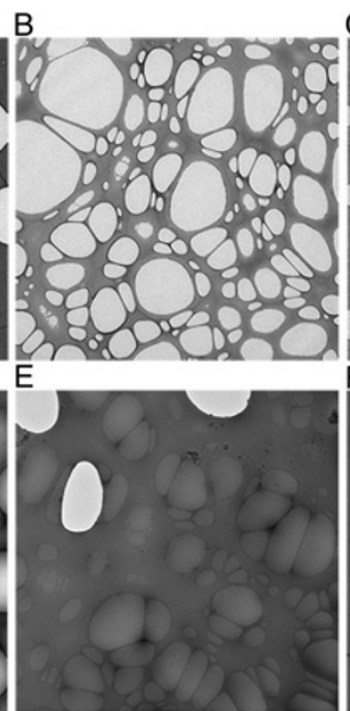

Lacey carbon grid

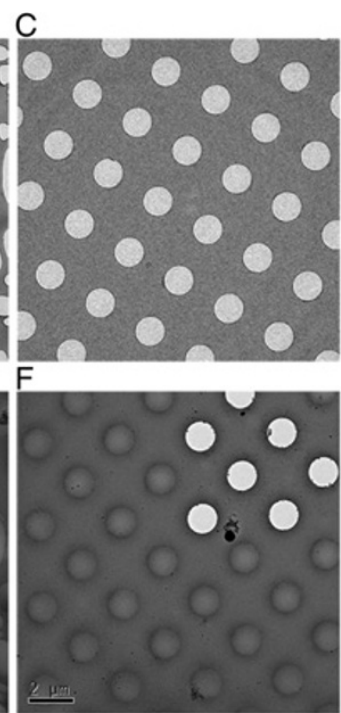

C-flat grid

Figure 1 TEM images of holey carbon grids in the absence and the presence of vitreous ice. (A-C) Apparent views of Cryo-EM grids used in this study: Quantifoil grid (A), Lacey carbon grid (B), C-flat grid (C). (D-F) Appearances of each grid type with embedded vitreous ice. Note that C-flat grid is manufactured without plastics, thus it is known to be ultra-flat compared to other types of carbon grids.

\section{Results and discussion}

In order to correlate the thickness of supporting carbon film and that of the resulting vitreous ice, thickness of carbon film with no vitreous ice was measured using EELS. Average (represented by standard deviation) of 20 independent measurements from different areas of each grid type was used for evaluation (Figures 1 and 2). It was found that Quantifoil grid had the thickest carbon $(49.11 \pm 8.50 \mathrm{~nm}$; mean \pm S.D. $)$ whereas $C$-flat grid had the thinnest $(17.32 \pm 0.82 \mathrm{~nm})$. Reported carbon film thickness of C-flat grid (10-20 nm) agreed well with reported experimental data (Quispe et al. 2007). Lacey carbon grid had carbon film with an intermediate thickness $(28.36 \pm 2.95 \mathrm{~nm})$. Variation of film thickness from different areas was minimal for C-flat grid (S.D. $0.82 \mathrm{~nm}$ ), suggesting the most uniform carbon film amongst the three types of grids tested.

Distilled water with no solute was subjected to the analysis since adsorption of extra materials onto the grids may introduce further variability to the outcome. Thickness of vitreous ice was measured from each type of grid that was prepared under the same vitrification condition (Figure 2). Each measurement was obtained from the entire hole without surrounding carbon film (Figure 2B). Average of 20 independent measurements was used for evaluation (Figure 2D). Ice thickness ranged from the largest to the smallest in the order of Quantifoil grid $(127.65 \pm 12.42 \mathrm{~nm})$, lacey carbon grid $(99.05 \pm 6.98 \mathrm{~nm})$ and C-flat grid $(92.35 \pm 6.37 \mathrm{~nm})$. In addition, ice thickness variation observed for Quantifoil grid (S.D. $12.42 \mathrm{~nm}$ ) was almost two fold higher than that of lacey carbon grid (S.D. $6.98 \mathrm{~nm}$ ) and C-flat grid (S.D. $6.73 \mathrm{~nm}$ ), suggesting the variation of carbon film thickness is directly reflected on the uniformity of resulting vitreous ice.

Variation of vitreous ice thickness across the hole was often apparent from electron micrographs, forming a smooth density gradient from the center toward the edge of the hole. Such thickness variation, so called 'lens effect', is well known for vitrified specimen, and may

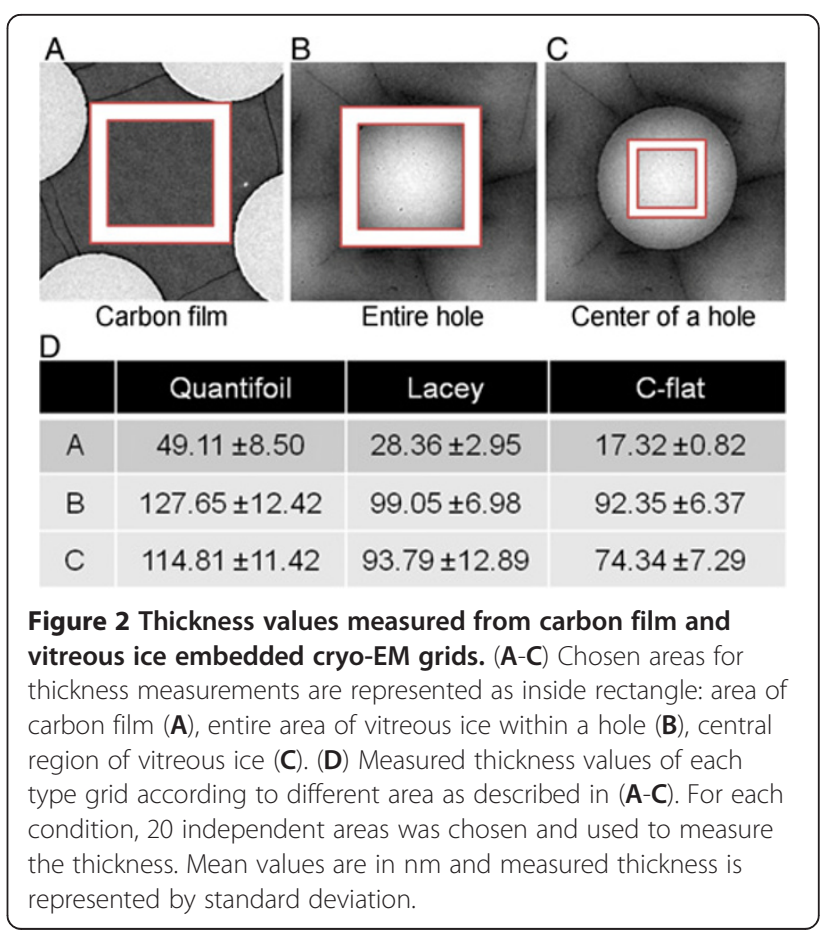


contribute to uneven particle distribution that can hamper efficient data collection. In order to characterize the lens effect for each grid type, micrographs were visually examined. All the grid types showed typical lens effect with varying extent although lacey carbon grid did not show distinctive, circular lens effect due to irregular size and shape of the holes (Figure 1).

For a more quantitative analysis, ice thickness of the center of a hole, which had the minimal electron density variation, was estimated (Figure $2 \mathrm{C}$ ). In consistent to bare carbon thickness and ice thickness of the entire hole, the thickness of the center of a hole varied from thickest to the thinnest in the order of Quantifoil grid $(114.81 \pm 11.42 \mathrm{~nm})$, lacey carbon grid $(93.79 \pm 12.89 \mathrm{~nm})$ and C-flat grid $(74.34 \pm 7.29 \mathrm{~nm})$. However, the extent of lens effect, as characterized by the difference between the ice thickness between the entire hole and the center, was most pronounced for C-flat grid $(18.01 \mathrm{~nm})$, followed by Quantifoil grid $(12.84 \mathrm{~nm})$ and lacey carbon grid $(5.26 \mathrm{~nm})$.

In this study we have used a simple method for estimating the thickness of vitreous ice using electron energy loss spectroscopy (EELS) and the log-ratio method. Thicknesses of supporting carbon film and embedded vitreous ice for three types of widely used holey carbon grids were efficiently measured. It was found that thickness of resulting vitreous ice was different in each type grid, possibly dependent of the thickness and of supporting carbon film. In addition, the extent of continuous variation of the ice thickness within the hole was characterized by estimating difference between the ice thickness of the entire hole and the central region.

Thickness of carbon support film and vitreous ice varied from thickest to thinnest in the order of Quantifoil grid, lacey carbon grid and C-flat grid. Substantial discrepancy between measured thickness of holy carbon grids from this study and that of manufacturer's description is possibly due to residual plastic layer underneath carbon film (Ermantraut et al. 1998), which may have hampered correct EELS measurement. Quantitative analysis showed that the lens effect was most pronounced for C-flat grid, followed by Quantifoil grid and lacey carbon grid. However, lacey carbon grid lacks symmetrically circular hole, and hence the thickness of the entire hole is unlikely to be estimated by using symmetric beam illumination.

Literatures suggest thinnest possible ice thickness that does not alter the integrity of the protein structure is optimal for cryo-EM (Orlova \& Saibil 2011). With this respect, control of ice thickness for a given sample is extremely important. For extreme cases, ice thickness of $700-800 \mathrm{~nm}$ is required as exemplified by cryo-EM study of giant Mimivirus (Xiao et al. 2005; Xiao et al. 2009), whereas the thinnest achievable ice thickness is necessary for $7 \mathrm{~nm}$ DNA tetrahedron (Kato et al. 2009). Therefore characterization of vitreous ice thickness shown in this study would be beneficial, along with other vitrification parameters, for obtaining optimal cryo-EM data.

\section{Abbreviations}

TEM: Transmission electron microscopy; NMR: Nuclear magnetic resonance; Cryo-EM: Cryo-electron microscopy; EELS: Electron energy loss spectroscopy; Lab6: Lanthanum hexaboride; GIF: Gatan imaging filter.

\section{Competing interests}

The authors declare that they have no competing interests.

\section{Authors' contributions}

H.S.J. designed research; H-J.C. and J-K.H. performed researches; J-G.K. contributed spectroscopy analysis. H-J.C. J-K.H. H.S.J. H.N.P. and D-J.Y. analzed EM data. J-K.H. and H.S.J. wrote the paper. All authors read and approved the final manuscript.

\section{Acknowledgements}

This work was supported by Korea Basic Science Institute grant (T33415) to JK Hyun.

Received: 12 March 2013 Accepted: 12 March 2013

Published: 18 April 2013

\section{References}

Frank J (2006) Three-dimensional electron microscopy of macromolecular assemblies: visualization of biological molecules in their native state, 2nd edition. Oxford University Press, New York

van Heel M, Gowen B, Matadeen R, Orlova EV, Finn R, Pape T, Cohen D, Stark H, Schmidt R, Schatz M, Patwardhan A (2000) Single-particle electron cryo-microscopy: towards atomic resolution. Q Rev Biophys 33:307-369

Adrian M, Dubochet J, Lepault J, McDowall AW (1984) Cryo-electron microscopy of viruses. Nature 308:32-36

Cavalier A, Spehner D, Humbel BM (2008) Handbook of cryo-preparation methods for electron microscopy. CRC Press, Boca Raton

Gonen T, Cheng Y, Sliz P, Hiroaki Y, Fujiyoshi Y, Harrison SC, Walz T (2005) Lipid-protein interactions in double-layered two-dimensional AQP0 crystals. Nature 438:633-638

Frank J, Radermacher M, Penczek P, Zhu J, Li Y, Ladjadj M, Leith A (1996) SPIDER and WEB: processing and visualization of images in $3 \mathrm{D}$ electron microscopy and related fields. J Struct Biol 116:190-199

van Heel M, Harauz G, Orlova EV, Schmidt R, Schatz M (1996) A new generation of the IMAGIC image processing system. J Struct Biol 116:17-24

Grigorieff N (2007) FREALIGN: high-resolution refinement of single particle structures. J Struct Biol 157:117-125

Orlova EV, Saibil HR (2011) Structural analysis of macromolecular assemblies by electron microscopy. Chem Rev 111:7710-7748

Aebi U, Pollard TD (1987) A glow discharge unit to render electron microscope grids and other surfaces hydrophilic. J Electron Microsc Tech 7:29-33

lancu CV, Tivol WF, Schooler JB, Dias DP, Henderson GP, Murphy GE, Wright ER, Li Z, Yu Z, Briegel A, Gan L, He Y, Jensen GJ (2006) Electron cryotomography sample preparation using the Vitrobot. Nat Protoc 1:2813-2819

Grassucci RA, Taylor D, Frank J (2008) Visualization of macromolecular complexes using cryo-electron microscopy with FEl Tecnai transmission electron microscopes. Nat Protoc 3:330-339

Malis T, Cheng SC, Egerton RF (1998) EELS log-ratio technique for specimen-thickness measurement in the TEM. J Electron Microsc Tech 8:193-200

Quispe J, Damiano J, Mick SE, Nackashi DP, Fellmann D, Ajero TG, Carragher B, Potter CS (2007) An improved holey carbon film for cryo-electron microscopy. Microsc Microanal 13:365-371

Ermantraut E, Wohlfart K, Tichelaar W (1998) Perforated support foils with predefined hole size, shape and arrangement. Ultramicroscopy 74:75-81 
Xiao C, Chipman PR, Battisti AJ, Bowman VD, Renesto P, Raoult D, Rossmann MG (2005) Cryo-electron microscopy of the giant Mimivirus. J Mol Biol 353:493-496

Xiao C, Kuznetsov YG, Sun S, Hafenstein SL, Kostyuchenko VA, Chipman PR, Suzan-Monti M, Raoult D, McPherson A, Rossmann MG (2009) Structural studies of the giant mimivirus. PLoS Biol 7:e92

Kato T, Goodman RP, Erben CM, Turberfield AJ, Namba K (2009) High-resolution structural analysis of a DNA nanostructure by cryoEM. Nano Lett 9:2747-2750

doi:10.1186/2093-3371-4-7

Cite this article as: Cho et al:: Measurement of ice thickness on

vitreous ice embedded cryo-EM grids: investigation of optimizing

condition for visualizing macromolecules. Journal of Analytical Science and Technology 2013 4:7.

\section{Submit your manuscript to a SpringerOpen ${ }^{\circ}$} journal and benefit from:

- Convenient online submission

- Rigorous peer review

- Immediate publication on acceptance

- Open access: articles freely available online

- High visibility within the field

- Retaining the copyright to your article

Submit your next manuscript at $>$ springeropen.com 